\title{
Connectedness of the Tannakian group attached to semi-stable multiple filtrations
}

\author{
MASAmi FuJimori
}

ABSTRACT - We show that the affine group scheme whose category of finite dimensional representations is equivalent to a tensor category of finite dimensional vector spaces equipped with semi-stable (multiple) filtrations of slope zero is connected.

Mathematics Subject Classification (2010). 20G05 (primary), 14L24, 18D10 (secondary).

KEYwords. Finite group, representation, semi-simple, slope, tensor category, unipotent.

\section{Introduction}

Let $K$ be an arbitrary field, $L$ a separable algebraic closure of $K$, and $M$ any set of indices. We denote by $\mathcal{C}(K, L, \mathfrak{M})$ the tensor category of finite dimensional vector spaces over $K$ equipped with multiple filtrations over $L$ indexed by the set $\mathfrak{M}$. We write $\mathcal{C}_{0}^{\mathrm{ss}}(K, L, \mathfrak{M})$ for the full subcategory of $\mathcal{C}(K, L, \mathfrak{M})$ composed of semi-stable objects of slope zero and of zero objects. We quickly recall the definitions [6, Definition $1.9 \&$ Definition 1.16] of $\mathcal{C}(K, L, \mathfrak{M})$ and $\mathcal{C}_{0}^{\text {ss }}(K, L, \mathfrak{M})$.

For a finite dimensional vector space $V$ over $K$, a family of subspaces $F^{i} V(i \in \mathbb{R})$ over $L$ of $L \otimes_{K} V$ is called a filtration over $L$ of $V$ if the relations

(*) Indirizzo dell'A.: Kanagawa Institute of Technology (Shimo-Ogino 1030, Atsugi, Kanagawa) 243-0292 Japan.

E-mail: fujimori@gen.kanagawa-it.ac.jp 


$$
\begin{array}{cc}
F^{i} V \supset F^{j} V(i \leq j), & \bigcup_{i \in \mathbb{R}} F^{i} V=L \otimes_{K} V, \\
\bigcap_{i \in \mathbb{R}} F^{i} V=0, & F^{i} V=\bigcap_{j<i} F^{j} V
\end{array}
$$

are enjoyed. An object of $\mathcal{C}(K, L, \mathfrak{M})$ is a finite dimensional vector space $V$ over $K$ equipped with a family of filtrations $F_{v} V(v \in \mathfrak{M})$ over $L$ of $V$ such that for except a finite number of indices $v$, the filtrations are trivial:

$$
F_{v}^{i} V=\left\{\begin{array}{cc}
L \otimes_{K} V & (i \leq 0) \\
0 & (i>0)
\end{array}\right.
$$

A morphism between two objects of $\mathcal{C}(K, L, \mathfrak{M})$ is a linear map over $K$ between the underlying vector spaces over $K$ which respects all their filtrations when linearly extended over $L$. For two objects $\left(V,\left(F_{v} V\right)_{v \in \mathfrak{M}}\right)$ and $\left(W,\left(F_{v} W\right)_{v \in \mathfrak{M}}\right)$, their tensor product is the vector space $V \otimes_{K} W$ over $K$ equipped with filtrations over $L$

$$
F_{v}^{i}\left(V \otimes_{K} W\right)=\sum_{j+q=i} F_{v}^{j} V \otimes_{L} F_{v}^{q} W \quad(i \in \mathbb{R}) .
$$

The category $\mathcal{C}(K, L, \mathfrak{M})$ thus defined is a $K$-linear additive tensor category.

For a non-zero object $\left(V,\left(F_{v} V\right)_{v \in \mathfrak{M}}\right)$ (below, we call it $V$ for short) of $\mathcal{C}(K, L, \mathfrak{M})$, the slope $\mu(V)=\mu\left(V,\left(F_{v}^{\cdot} V\right)_{v \in \mathfrak{M}}\right)$ is a real number given by

$$
\mu(V)=\sum_{v \in \mathfrak{M}} \frac{1}{\operatorname{dim}_{K} V} \sum_{w \in \mathbb{R}} w \operatorname{dim}_{L} \operatorname{gr}^{w}\left(F_{v} V\right),
$$

where $\operatorname{gr}^{w}\left(F_{v} V\right)=F_{v}^{w} V / F_{v}^{w+} V, F_{v}^{w+} V=\cup_{j>w} F_{v}^{j} V$. A non-zero object $V$ of $\mathcal{C}(K, L, \mathfrak{M})$ is semi-stable if it satisfies the condition that for any monomorphism $W \rightarrow V$ in $\mathcal{C}(K, L, \mathfrak{M})$ of a non-zero object $W$, we have $\mu(W) \leq \mu(V)$.

A fundamental fact about the full subcategory $\mathcal{C}_{0}^{\mathrm{ss}}(K, L, \mathfrak{M})$ of $\mathcal{C}(K, L, \mathfrak{M})$ consisting of semi-stable objects of slope zero and of zero objects is the following:

Theorem 1.1 (Faltings [4], Totaro [7], cf. André [1]). Let $\omega_{0}^{\mathrm{ss}}(K, L, \mathfrak{M})$ be the forgetful functor of $\mathcal{C}_{0}^{\mathrm{SS}}(K, L, \mathfrak{M})$ onto the tensor category of finite dimensional vector spaces over $K$. The category $\mathcal{C}_{0}^{\mathrm{ss}}(K, L, \mathfrak{M})$ is canonically equivalent to the tensor category offinite dimensional representations over $K$ of an affine group scheme Aut $\omega_{0}^{\mathrm{ss}}(K, L, \mathfrak{M})$ over $K$ of natural equivalences of the functor $\omega_{0}^{\mathrm{ss}}(K, L, \mathfrak{M})$. 
In our previous paper [6], we have particularly shown that any connected reductive group over $K$ appears (up to isomorphism) in many ways as a quotient group scheme of the affine group scheme Aut $\omega_{0}^{\mathrm{ss}}(K, L, \mathfrak{M})$ when the cardinality of $\mathfrak{M}$ is infinite. Denoting by $\omega_{K}(G)$ the forgetful tensor functor of the tensor category $\operatorname{Rep}_{K}(G)$ of finite dimensional representations over $K$ of an affine group scheme $G$ over $K$ onto the tensor category of finite dimensional vector spaces over $K$, this result is differently stated that when $G$ is connected reductive, there exists a fully faithful tensor functor $\imath$ of $\operatorname{Rep}_{K}(G)$ into $\mathcal{C}_{0}^{\mathrm{sS}}(K, L, \mathfrak{M})$ such that $\omega_{0}^{\mathrm{ss}}(K, L, \mathfrak{M}) \circ \imath=\omega_{K}(G)$.

In our present paper, we prove the next:

THEOREM 1.2. Let l be any tensor functor of $\operatorname{Rep}_{K}(G)$ to $\mathcal{C}_{0}^{\mathrm{sS}}\left(K, L, \mathfrak{M}_{\mathrm{C}}\right)$ such that $\omega_{0}^{\mathrm{sS}}(K, L, \mathfrak{M}) \circ \imath=\omega_{K}(G)$. If the group scheme $G$ over $K$ is finite, then for any object $V$ of $\operatorname{Rep}_{K}(G)$, the image $(V)$ must be an object with all the filtrations trivial. In particular, the functor $\mathrm{c}$ cannot be full unless $G=1$.

Since the group scheme $\pi_{0}\left(\right.$ Aut $\left.\omega_{0}^{\mathrm{ss}}(K, L, \mathfrak{M})\right)$ of connected components of the affine group scheme Aut $\omega_{0}^{\mathrm{ss}}(K, L, \mathfrak{M})$ is pro-étale (cf., e.g., [3, III, § 3, 7.7]), our theorem implies the following:

Corollary 1.3. The affine group scheme Aut $\omega_{0}^{\mathrm{ss}}(K, L, \mathfrak{M})$ over $K$ is connected.

In Section 2, a proof of Theorem 1.2 is given for an arbitrary (finite or infinite) GaLOIS extension $L$. It might be useful and natural to bring in a finite étale $K$-algebra $L$. But we would like in the present paper to stick to our original setting $[5,6]$ bearing Diophantine approximation in mind. A little deviation is that the index set $\mathfrak{M}$ is any non-empty set. In Section 3, we make a few observations on the problem to find all (algebraic) quotients of Aut $\omega_{0}^{\mathrm{ss}}(K, L, \mathfrak{M})$.

\section{Finite groups}

Let $K$ be an arbitrary field, $G$ a finite group scheme over $K$, and $K[G]$ the $K$-algebra of global functions on $G$. Note that as $G$ is finite over $K$, the group scheme $G$ is affine (over $K$ ). The key to the result of the present paper is that the dual vector space $K[G]^{*}$ over $K$ of $K[G]$ is a finite dimensional representation of $G$ by (left) translation. An immediate wellknown consequence is the following: 
LEMMA 2.1. Each finite dimensional representation over $K$ of $G$ is (canonically) a quotient representation of a finite direct sum of copies of $K[G]^{*}$.

We recall its proof for the convenience of readers.

Proof. Let $V$ be a finite dimensional representation over $K$ of $G$. By the very definition of representation, the comorphism

$$
V^{*} \hookrightarrow K[G] \otimes_{K} V^{*}
$$

of the action of $G$ on $V$ is an injective comorphism between representation spaces when the tensor product $K[G]^{*} \otimes_{K} V$ is regarded as a representation with the trivial action on $V$. This tells us that the representation $V$ is a quotient of $K[G]^{*} \otimes_{K} V$ which is isomorphic to a finite direct sum of copies of $K[G]^{*}$.

Let $L$ be a (finite or infinite) GaLoIs extension field of $K$ and $\mathfrak{M}$ any non-empty set of indices. For finite dimensional vector spaces over $K$ equipped with descending exhaustive separated left-continuous (multiple) filtrations defined over $L$ (called (multiple) filtrations over $L$ for simplicity [2][6], cf. (1) in Section 1), we consider the following quantities:

DeFINITION 2.2. Let $V$ be a finite dimensional non-zero vector space over $K$ equipped with a family of filtrations $F_{v} V$ over $L$ indexed by $\mathfrak{M}$. Writing gr for the graduation derived from a filtration, we set for all $v \in \mathfrak{M}$

$$
m_{v}(V)=\min \left\{w \in \mathbb{R} \mid \operatorname{gr}^{w}\left(F_{v} V\right) \neq 0\right\} .
$$

Remember that when saying multiple filtrations, we are tacitly assuming for except a finite number of indices $v \in \mathfrak{M}$, the filtrations $F_{v} V$ are trivial in the sense of (2) in Section 1. In particular, we see that $m_{v}(V)=0$ for almost all $v \in \mathfrak{M}$. We put

$$
m(V)=\sum_{v \in \mathfrak{M}} m_{v}(V) .
$$

REMARK 2.3. By the definition of slopes $\mu_{v}(v \in \mathfrak{M})$ of filtrations [6, Definition 1.12], we have for each $v \in \mathfrak{M}$

$$
m_{v}(V) \leq \frac{1}{\operatorname{dim}_{K} V} \sum_{w \in \mathbb{R}} w \operatorname{dim}_{L} \operatorname{gr}^{w}\left(F_{v} V\right)=\mu_{v}(V)
$$


and

$$
m(V) \leq \sum_{v \in \mathfrak{M}} \mu_{v}(V)=\mu(V) .
$$

For finite dimensional vector spaces $V$ and $W$ over $K$ equipped with multiple filtrations over $L$ indexed by $\mathfrak{M}$, the direct sum of filtrations was defined as

$$
F_{v}^{i}(V \oplus W)=F_{v}^{i} V \oplus F_{v}^{i} W \quad(i \in \mathbb{R}, v \in \mathfrak{M})
$$

We obtain

$$
m_{v}(V \oplus W)=\min \left\{m_{v}(V), m_{v}(W)\right\} \quad(v \in \mathfrak{M})
$$

hence

$$
m_{v}(V \oplus \cdots \oplus V)=m_{v}(V)
$$

From the definition of the tensor product of filtrations ((3) in Section 1), we get

$$
m_{v}(V \otimes W)=m_{v}(V)+m_{v}(W) \quad(v \in \mathfrak{M})
$$

in particular,

$$
m_{v}\left(V^{\otimes n}\right)=n \cdot m_{v}(V)
$$

LEMMA 2.4. Let $V$ and $W$ be finite dimensional non-zero vector spaces over $K$ equipped with multiple filtrations over $L$ indexed by $\mathfrak{M}$. If there is a filtered homomorphism of $V$ to $W$ which is surjective as a linear map between vector spaces, then we have $m_{v}(V) \leq m_{v}(W)$.

Proof. Call $f$ the filtered homomorphism in the statement. By the definition of filtered homomorphism, we have

$$
f\left(L \otimes_{K} V\right)=f\left(F_{v}^{i} V\right) \subset F_{v}^{i} W \subset L \otimes_{K} W \quad\left(i \leq m_{v}(V)\right) .
$$

Since $f$ is surjective, we see

$$
F_{v}^{i} W=L \otimes_{K} W \quad\left(i \leq m_{v}(V)\right),
$$

which means $m_{v}(W) \geq m_{v}(V)$.

Let $\operatorname{Rep}_{K}(G)$ be the tensor category of finite dimensional representations over $K$ of $G, \operatorname{Vec}_{K}$ the tensor category of finite dimensional vector 
spaces over $K$, and $\mathcal{C}_{0}^{\text {ss }}(K, L, \mathfrak{M})$ the tensor category of finite dimensional vector spaces over $K$ equipped with semi-stable multiple filtrations over $L$ indexed by $\mathfrak{M}$ of slope zero ([6, Definition 1.16], cf. Section 1$)$. We denote respectively by $\omega_{K}(G)$ and by $\omega_{0}^{\mathrm{ss}}(K, L, \mathfrak{M})$ the forgetful tensor functors of $\operatorname{Rep}_{K}(G)$ and of $\mathcal{C}_{0}^{\text {ss }}(K, L, \mathfrak{M})$ to $\operatorname{Vec}_{K}$.

Proof of Theorem 1.2 (for general $L$ ). We may suppose $V$ is not zero. Let $n$ be an arbitrary positive integer. By Lemma 2.1, there exists a surjective $G$-homomorphism $f$ onto the $n$-times tensor product $V^{\otimes n}$ of $V$ of a $G$-representation $W$ which is isomorphic to a finite direct sum of copies of $K[G]^{*}$. The assumption $\omega_{0}^{\mathrm{sS}}(K, L, \mathfrak{M}) \circ \imath=\omega_{K}(G)$ says that the morphism $l(f)$ as a linear map between vector spaces is $f$ itself. Due to Lemma 2.4, we see $m_{v}(W) \leq m_{v}\left(V^{\otimes n}\right)$. On the other hand, thanks to (5) and (6), we have $m_{v}(W)=m_{v}\left(K[G]^{*}\right)$ and $m_{v}\left(V^{\otimes n}\right)=n \cdot m_{v}(V)$. Hence we get

$$
\frac{1}{n} m_{v}\left(K[G]^{*}\right) \leq m_{v}(V) .
$$

Making $n$ large, we obtain

$$
0 \leq m_{v}(V) .
$$

As $\mu(V)=0$ by the definition of $\imath$, Remark 2.3 forces

$$
m(V)=\mu(V),
$$

which is possible only when all the filtrations are trivial.

REMARK 2.5. In general, the condition $m(V)=\mu(V)$ is not sufficient to assure that the filtrations are trivial. For example, let $\mathfrak{M}=\{0, \infty\}$, $V=K$,

$$
F_{0}^{i} V=\left\{\begin{array}{cc}
L \otimes_{K} V & (i \leq-1) \\
0 & (i>-1)
\end{array},\right.
$$

and

$$
F_{\infty}^{i} V=\left\{\begin{array}{cc}
L \otimes_{K} V & (i \leq 1) \\
0 & (i>1)
\end{array} .\right.
$$

We have $m_{0}(V)=\mu_{0}(V)=-1, m_{\infty}(V)=\mu_{\infty}(V)=1$, and $m(V)=\mu(V)=0$. The point of the proof of THEOREM 1.2 is that we can show $m_{v}(V) \geq 0$ for all $v \in \mathfrak{M}$. 


\section{Algebraic groups which do not or do appear}

Let $K$ be an arbitrary field, $L$ a (finite or infinite) GaLoIs extension, and $\mathfrak{M}$ any non-empty set of indices. We consider in this section a connected linear algebraic group $G$ over $K$ in general.

Proposition 3.1. Let $l$ be any tensor functor of $\operatorname{Rep}_{K}(G)$ to $\mathcal{C}_{0}^{\mathrm{ss}}(K, L, \mathfrak{M})$ such that $\omega_{0}^{\mathrm{sS}}(K, L, \mathfrak{M}) \circ \imath=\omega_{K}(G)$. If the group scheme $G$ over $K$ is unipotent, then for any object $V$ of $\operatorname{Rep}_{K}(G)$, the image $\imath(V)$ must be an object with all the filtrations trivial.

Proof. Denote by $U$ a one-dimensional trivial representation space over $K$ of $G$. Since $\imath(U) \otimes \imath(U) \simeq \imath(U \otimes U) \simeq l(U)$, the filtrations of $l(U)$ must be trivial, i.e., unit objects go to unit objects.

Let $V$ be any non-zero finite dimensional representation over $K$ of $G$. On the assumption that $G$ is unipotent, there exists an injective $G$-homomorphism of $U$ into $V$. The other assumption $\omega_{0}^{\mathrm{ss}}(K, L, \mathfrak{M}) \circ \iota=\omega_{K}(G)$ implies that the functor $l$ sends kernels to kernels and cokernels to cokernels (cf. [5, Lemma 1.8]). We have particularly an exact sequence

$$
0 \rightarrow \imath(U) \rightarrow \imath(V) \rightarrow \imath(V / U) \rightarrow 0 .
$$

When the filtrations of $\imath(V / U)$ are trivial, we see readily that those of $\imath(V)$ are also trivial. By the induction on the dimensions of representation spaces, we are done.

COROLlary 3.2. If $G$ is isomorphic to a quotient of the affine group scheme Aut $\omega_{0}^{\mathrm{ss}}(K, L, \mathfrak{M})$, then semi-simple elements generate a dense subgroup of $G$.

Proof. Let $N$ be the (ZARISKI) closure of the subgroup generated by semi-simple elements of $G$. The variety $N$ is a (closed) normal subgroup defined over $K$ of $G$. By definition, all the elements of the quotient group $G / N$ are unipotent, hence $G / N$ is unipotent. Since $G / N$ is isomorphic to a quotient of Aut $\omega_{0}^{\mathrm{ss}}(K, L, \mathfrak{M})$, Proposition 3.1 means that $G / N=1$.

REMARK 3.3. When $L$ is a separable closure of the base field $K$ and $\mathfrak{M}$ is infinite, we have shown in the appendix of our previous paper [6] that any affine algebraic group scheme a dense subgroup of which is generated by tori defined over $K$ appears (up to isomorphism) as a quotient of Aut $\omega_{0}^{\mathrm{ss}}(K, L, \mathfrak{M})$. Applying the method of [6] to tori defined 
over $L$, we observe that any affine algebraic group which fills the necessity in Corollary 3.2 really appears (up to isomorphism) as a quotient of Aut $\omega_{0}^{\mathrm{ss}}(K, L, \mathfrak{M})$. In this way, Corollary 3.2 presents a kind of characterization for linear algebraic groups which can be isomorphic to quotients of Aut $\omega_{0}^{\mathrm{ss}}(K, L, \mathfrak{M})$.

REMARK 3.4. Let $S$ be the linear algebraic group of upper triangular matrices of degree 2 with determinant 1 . The group $S$ is defined over an arbitrary field $K$, solvable, and generated by two split tori. According to [6, Theorem A.14], the solvable group $S$ appears (up to isomorphism) as a quotient of Aut $\omega_{0}^{\mathrm{ss}}(K, L, \mathfrak{M})$ provided at least the cardinality of the index set $\mathfrak{M}$ is greater than three. Thus the affine group scheme Aut $\omega_{0}^{\mathrm{ss}}(K, L, \mathfrak{M})$ is not pro-reductive and the Tannakian category $\mathcal{C}_{0}^{\mathrm{ss}}(K, L, \mathfrak{M})$ is not poly-stable in that case.

On the other hand, if the cardinality of $\mathfrak{M}$ is one, then the group $S$ cannot be isomorphic to any quotient of Aut $\omega_{0}^{\mathrm{ss}}(K, L, \mathfrak{M})$, because the onedimensional multiplicative group $\mathrm{G}_{\mathrm{m}}$ is isomorphic to a quotient of $S$ but one-dimensional objects of $\mathcal{C}_{0}^{\mathrm{SS}}(K, L, \mathfrak{M})$ are all units in this case.

Acknowledgments. The author expresses his gratitude to THE REFEREE for valuable suggestions and comments on the first version of the present paper.

\section{REFERENCES}

[1] Y. ANDRÉ, On nef and semistable hermitian lattices, and their behaviour under tensor product. Tôhoku Math. J., 63(4): pp. 629-649, 2011.

[2] J.-F. DAT - S. ORLIK - M. RAPOPORT, Period Domains over Finite and p-adic Fields, volume 183 of Cambridge Tracts in Math. Cambridge Univ. Press, New York, 2010.

[3] M. Demazure - P. Gabriel, Groupes Algébriques, volume 1. North-Holland Publishing Company, Amsterdam, The Netherlands, 1970.

[4] G. Faltings, Mumford-Stabilität in der algebraischen Geometrie. In Proceedings of the International Congress of Mathematicians, Zürich, Switzerland 1994, pp. 648-655, Basel, Switzerland, 1995. Birkhäuser Verlag.

[5] M. Fujimori, On systems of linear inequalities. Bull. Soc. Math. France, 131(1): pp. 41-57, 2003. Corrigenda. ibid., 132(4): pp. 613-616, 2004.

[6] M. FuJIMORI, The algebraic groups leading to the Roth inequalities. J. Théor. Nombres Bordeaux, 24: pp. 257-292, 2012.

[7] B. Totaro, Tensor products in p-adic Hodge theory. Duke Math. J., 83: pp. 79-104, 1996.

Manoscritto pervenuto in redazione il 13 Febbraio 2013. 